\title{
IRAS F02044+0957: A Radio Source in an Interacting System of Galaxies
}

\author{
O. V. Verkhodanov
}

Special Astrophysical Observatory, Nizhnij Arkhyz, Russia

\author{
V. H. Chavushyan, R. Mújica and J. R. Valdés \\ Instituto Nacional de Astrofísica Óptica y Electrónica, Puebla, México
}

\section{S. A. Trushkin}

Special Astrophysical Observatory RAS, Nizhnij Arkhyz, Russia

\begin{abstract}
The steep spectrum of IRAS F02044+0957 was obtained with the RATAN-600 radio telescope at four frequencies. Optical spectroscopy of the system components was carried out with the $2.1 \mathrm{~m}$ telescope of the Guillermo Haro Observatory. The observational data allow us to conclude that this object is a pair of interacting galaxies, a LINER and an $\mathrm{H}$ II galaxy, at $z=0.093$.
\end{abstract}

Trushkin \& Verkhodanov (1995) compiled a list of about 750 objects by using the CATS, as a result of cross-identifications of infrared IRAS catalogues and the source catalogue of the Texas survey at $365 \mathrm{MHz}$. From the sample of steep spectrum sources we selected those without classification in public databases (CATS, NED, ADS and LEDA). One of these objects is IRAS F02044+0957, identified with the NVSS radio source J020706 +101147 .

Radio observations were carried out on April 23-25, 1999, with the North Sector of the RATAN-600 telescope. The wide-band radiometer complex was used at four frequencies: $2.3,3.9,7.7$ and $11.2 \mathrm{GHz}$. The nearby non-variable radio source PKS $1345+12$ was used as the flux density calibration source. A linear fitting to the radio spectrum was used to estimate a spectral index by the least squares method. Each point of the spectrum was weighted proportionally to the value of $1 /\left(\Delta S / S_{\nu}\right)^{2}$; where $\Delta S / S_{\nu}$ is the relative error of the flux density. The radio spectrum is described by a power-law $S_{\nu}[J y]=85 \nu^{-0.94 \pm 0.02} \mathrm{MHz}$. The radio luminosity in the frequency interval from 365 to $10^{4} \mathrm{MHz}$ is $L=$ $9.381 \times 10^{34} \mathrm{erg} \mathrm{sec}^{-1} \mathrm{~cm}^{-2}$ (assuming $H_{0}=64 \mathrm{~km} \mathrm{Mpc}^{-1} \mathrm{sec}^{-1}$ and $q_{0}=0.8$ ).

Optical spectroscopy of the objects marked by A, B, C, D, and E in Fig. 1 was obtained in August and November 1999. We used the 2.1m telescope of the Guillermo Haro Observatory (GHO) in Cananea, Sonora, Mexico, operated by the National Institute of Astrophysics, Optics and Electronics (INAOE). The Faint Object Spectrograph and Camera (LFOSC) (Zickgraf et al. 1997) was used. A setup, covering the spectral range $4200-9000 \AA$ with a dispersion of $8.2 \AA /$ pix was adopted. The effective instrumental spectral resolution is about $15 \AA$. 

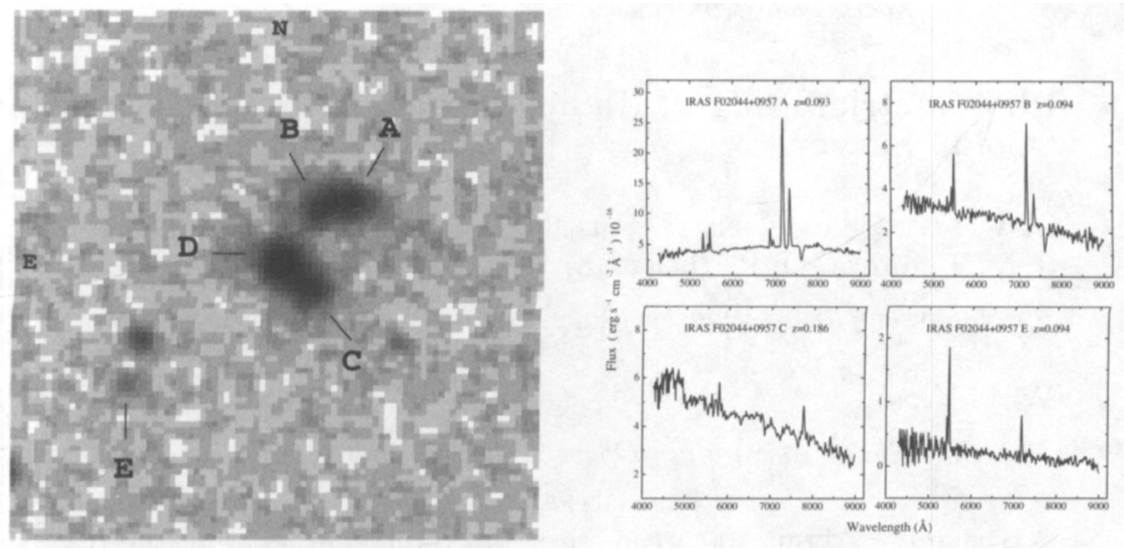

Figure 1. DSS image of IRAS F02044+0957 and corresponding spectra

The data reduction was done using the IRAF packages and included bias and flat field corrections, cosmic rays cleaning, wavelength linearization and flux transformation.

The detailed spectroscopy showed that objects A and B are apparently a pair of interacting emission-line galaxies at $z=0.093$. The second pair (objects $\mathrm{C}$ and $\mathrm{D}$ ) is a geometrical projection of a star (D) and an emission line galaxy (C) at $\mathrm{z}=0.186$. It is not possible that the G type Main Sequence star, is the source of infrared and radio emission.

IRAS F02044+0957 is located at $16^{\prime}$ from the center of the galaxy cluster ZwCL $0203.6+1008$ (Zwicky 619). The radius of the cluster is 12.6 . This means that IRAS F02044+0957 must be out the cluster boundaries.

In order to investigate the nature of the interacting system $\mathrm{AB}$, we used the diagnostic diagrams $\log ([\mathrm{N} \mathrm{II}] \lambda 6583 / \mathrm{H} \alpha)$ vs $\log ([\mathrm{O} \mathrm{III}] \lambda 5007 / \mathrm{H} \beta)$ and $\log ([\mathrm{S} \mathrm{II}]-$ $\lambda \lambda 6717+6731 / \mathrm{H} \alpha)$ vs $\log ([\mathrm{O}$ III $] \lambda 5007 / \mathrm{H} \beta)$.

The values for component $\mathrm{A}, \log ([\mathrm{N} \mathrm{II}] / \mathrm{H} \alpha)=-0.15$ and $\log ([\mathrm{SII}] / \mathrm{H} \alpha)=$ -0.23 , put the object on both diagrams in the AGN region, very close to the boundary with the $\mathrm{H}$ II region-like galaxies. According to the criteria for spectral classification proposed by Ho, Filippenko \& Sargent (1997) (HFS97), the component $\mathrm{A}$ is a LINER because the value of $\left[\mathrm{O}_{\mathrm{I}}\right] \lambda 6300 / \mathrm{H} \alpha=0.26$. For component $\mathrm{B}$, we obtained the values: $\log ([\mathrm{N} \mathrm{II}] / \mathrm{H} \alpha)=-0.76$ and $\log ([\mathrm{S} \mathrm{II}] / \mathrm{H} \alpha)=-0.55$. Therefore, according to HFS97, the component B is a H II galaxy. Summarizing, the interacting system $\mathrm{AB}$ is composed of a LINER and an $\mathrm{H}$ II galaxy.

Acknowledgments. This work was partially supported by CONACyT grants 28499-E, J32178-E, and 32106-E.

\section{References}

Heckman, T. M. 1980, A\&A, 87, 152

Ho, L. C., Filippenko, A. V., Sargent, W. L. W. 1997, ApJS, 112, 315

Sanders, D. B., Soifer, B. T., Elias, J. H., et al., 1988, ApJ, 325, 74

Trushkin, S. A., Verkhodanov, O. V. 1995, Bulletin of SAO, 39, 150.

Zickgraf, F. J., Thiering, I., Krautter, J., et al., 1997, A\&AS, 123, 103. 